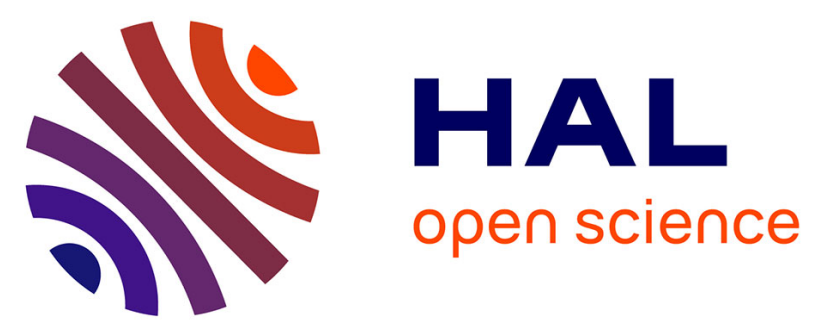

\title{
Non-linear elastodynamic analysis by the BEM: an approach based on the iterative coupling of the D-BEM and TD-BEM formulations
}

Delfim Soares, Jose Antonio Marques Carrer, Webe João Mansur

\section{- To cite this version:}

Delfim Soares, Jose Antonio Marques Carrer, Webe João Mansur. Non-linear elastodynamic analysis by the BEM: an approach based on the iterative coupling of the D-BEM and TDBEM formulations. Engineering Analysis with Boundary Elements, 2005, 29 (8), pp.761 - 774. 10.1016/j.enganabound.2005.04.005 . hal-01634270

\author{
HAL Id: hal-01634270 \\ https://hal.science/hal-01634270
}

Submitted on 14 Nov 2017

HAL is a multi-disciplinary open access archive for the deposit and dissemination of scientific research documents, whether they are published or not. The documents may come from teaching and research institutions in France or abroad, or from public or private research centers.
L'archive ouverte pluridisciplinaire HAL, est destinée au dépôt et à la diffusion de documents scientifiques de niveau recherche, publiés ou non, émanant des établissements d'enseignement et de recherche français ou étrangers, des laboratoires publics ou privés. 


\title{
Non-linear elastodynamic analysis by the BEM: an approach based on the iterative coupling of the D-BEM and TD-BEM formulations
}

\author{
D. Soares Jr. ${ }^{\text {a }}$, J.A.M. Carrer ${ }^{\mathrm{b}}$, W.J. Mansur ${ }^{\mathrm{a}, *}$ \\ ${ }^{\mathrm{a}}$ COPPE-Federal University of Rio de Janeiro, Civil Engineering Department, CP 68506, CEP 21945-970, Rio de Janeiro, RJ, Brazil \\ bPPGMNE: Programa de Pós-Graduação em Métodos Numéricos em Engenharia, Universidade Federal do Paraná, Caixa Postal 19011, \\ CEP 81531-990, Curitiba, PR, Brasil
}

The present paper is concerned with the development of a scheme based on iterative coupling of two boundary element formulations to obtain time-domain numerical solution of dynamic non-linear problems. The domain is divided into two sub-domains: the sub-domain that presents non-linear behaviour is modelled by the D-BEM formulation (D: domain) whereas the sub-domain that behaves elastically is modelled by the TD-BEM formulation (TD, time-domain). The solution of the problem is obtained independently in each sub-domain and the variables at common interfaces are computed iteratively. Two examples are presented, in order to verify the potentialities of the proposed methodology.

Keywords: D-BEM formulation; TD-BEM formulation; Iterative coupling; Dynamic non-linear analysis; Time-domain BEM

\section{Introduction}

In this paper an approach based on the boundary element method (BEM) is presented and discussed in detail. Two BEM formulations, namely the so-called D-BEM (D stands for domain) and the TD-BEM (TD stands for time-domain) are employed in order to perform 2D non-linear elastodynamic analyses.

The D-BEM formulation employs the static fundamental solution (Kelvin fundamental solution) and keeps, in the BEM integral equations, the domain integral related to the inertial terms, i.e. the domain integral related to the acceleration. As a consequence, in order to perform dynamic analyses, the entire domain has to be discretized and time marching schemes, similar to those employed by FEM based approaches, may be adopted in order to advance in time (as usual, FEM means finite element method). The necessity of domain discretization does not turn this formulation unattractive. As pointed

\footnotetext{
* Corresponding author. Tel:: +55 2125627382.

E-mail address: webe@coc.ufrj.br (W.J. Mansur).
}

out by Telles [1], when performing elastoplastic analyses, the part of the domain where inelastic behaviour is expected to occur necessarily requires domain discretization. This feature, added to the simplicity of the D-BEM formulation, turns it very attractive and a natural choice to non-linear elastodynamic analyses [2]. It is important to notice that the DR-BEM (DR means dual reciprocity), in which the inertial domain integral is transformed into a boundary integral by a suitable choice of interpolation functions, can also be used in the approach discussed here as an alternative to the D-BEM. DR-BEM received a great deal of attention during the last years $[3,4]$. One important aspect related to the D-BEM formulation (and, also, to the DR-BEM) that deserves attention is concerned with time-marching: among the various timemarching methods available, the Houbolt scheme, based on a Lagrange polynomial of the third degree [5] have been widely employed. Therefore, the search for others and, perhaps, more adequate schemes (in other words, why a time-marching scheme fails completely and another one can be used successfully?) seems to be a very promising area of research. Recently, Carrer and Mansur [6] and Souza et al. [7] presented two papers concerning this matter. It is important to point out one important limitation of the D-BEM formulation (and also 
of the DR-BEM): as the radiation condition is not fulfilled by the fundamental solution, infinite domains cannot be adequately modelled, that is, in the analysis of an infinity medium, the interruption of the cells mesh creates an artificial boundary that produces undesirable reflection of the waves arriving at it. The artificial reflection tends to invalidate the numerical results once artificial waves reach the region of interest.

TD-BEM formulations, on the other hand, employ timedependent fundamental solutions. The use of an appropriate fundamental solution turns this formulation very elegant and attractive from the mathematical point of view [8-10]. Besides, the fulfilment of the radiation condition by the fundamental solution turns this formulation very suitable for performing infinite domain analysis, since there are no reflected waves from infinity. Reliable and accurate results are obtained; however, one must pay a price: instead of an inertial domain integral, convolution integrals need to be evaluated from the beginning of the analysis up to the current time. As convolution is an expensive process when late time results are required, the development of special techniques to reduce computational costs has become an important task. The truncation strategy used here to deal with this matter leads to an algorithm that makes unnecessary the assemblage of all matrices generated during the time-stepping process and required to compute the convolution integral inherent to the TD-BEM approach. Thus, it is possible to keep a compromise between the accuracy of the TD-BEM formulation and the computational efficiency [11].

Due to the characteristics of the two BEM formulations mentioned above, it looks natural and straightforward to employ, in a non-linear elastodynamic analysis, the D-BEM formulation for the part of the domain in which inelastic behaviour is expected to occur and the TD-BEM formulation for the part of the domain that behaves elastically (along the text, these sub-domains will be referred to simply as D-BEM sub-domain and TD-BEM sub-domain, respectively). In an infinite domain analysis, the interface between the non-linear and the linear domains, that is, between the D-BEM sub-domain and the TD-BEM sub-domain, can be interpreted as an efficient non-reflecting boundary.

It is important to mention that in BEM dynamic analyses, the correct choice of the time-step length plays a fundamental role. As the time-step lengths required by the D-BEM to produce reliable results are usually smaller than those required by the TD-BEM, special time/space interpolation/extrapolation procedures are employed, which turns out to be very effective and easy to implement in an iterative coupling approach. The proposed final algorithm is very powerful, as demonstrated by the examples presented at the end of this paper.

The paper contents are described next: the TD-BEM formulation is presented in Section 2; Section 3 is concerned with the D-BEM formulation, the time-marching scheme, the non-linear initial stress approach, and the solution techniques; in Section 4 the TD- and D-BEM iterative coupling procedure is described; Section 5 presents the numerical examples and Section 6 is dedicated to the conclusions of the paper.

\section{TD-BEM formulation}

The main aspects of the TD-BEM formulation will be presented below. For additional details, the reader is referred to $[9,10]$.

In the absence of initial conditions, the basic integral equation of the TD-BEM approach for two-dimensional elastodynamics can be written as (see Fig. 2b-d):

$$
\begin{aligned}
C_{i k}(\xi) u_{k}(\xi ; t)= & \int_{\Gamma} \int_{0}^{t^{+}} u_{i k}^{*}(X, t ; \xi, \tau) p_{k}(X, \tau) \mathrm{d} \tau \mathrm{d} \Gamma(X) \\
& -\iint_{\Gamma}^{t^{+}} p_{i k}^{*}(X, t ; \xi, \tau) u_{k}(X, \tau) \mathrm{d} \tau \mathrm{d} \Gamma(X)
\end{aligned}
$$

In Eq. (1) the following notation is adopted: in the inner integral, $t$ represents the final analysis time and $\tau$ is the time integration variable; in the outer integral, $\Gamma$ represents the boundary; $u_{k}(X, \tau)$ and $p_{k}(X, \tau)$ are the displacement and traction components in the $k$-direction; $u_{i k}^{*}(X, t ; \xi, \tau)$ is the fundamental solution and $p_{i k}^{*}(X, t ; \xi, \tau)$ may be called the fundamental traction. Additionally, the elastodynamic fundamental solution, that represents the displacement component in the $k$-direction at the field point $X$ corresponding to a unit impulse applied in the $i$ direction at the source point $\xi$ at the time $\tau$, can be written as:

$$
\begin{aligned}
& 2 \pi \rho c_{\mathrm{s}} u_{i k}^{*}(X, t ; \xi, \tau) \\
& =\left(E_{i k} L_{\mathrm{s}}+F_{i k} L_{\mathrm{s}}^{-1}+J_{i k} N_{\mathrm{s}} L_{\mathrm{s}}\right) H_{\mathrm{s}}-\frac{c_{\mathrm{s}}}{c_{\mathrm{d}}}\left(F_{i k} L_{\mathrm{d}}^{-1}\right. \\
& \left.\quad+J_{i k} L_{\mathrm{d}} N_{\mathrm{d}}\right) H_{\mathrm{d}}
\end{aligned}
$$

The tensors $E_{i k}, F_{i k}, J_{i k}$ are given by

$E_{i k}=\delta_{i k}$

$F_{i k}=\frac{\delta_{i k}}{r^{2}}$

$J_{i k}=\frac{r_{,} r_{, k}}{r^{2}}$

where $\delta_{i k}$ is the Kronecker delta and $r=r(\xi, X)$ is the distance between $X$ and $\xi$. Furthermore:

$r=\left(r_{i} r_{i}\right)^{1 / 2} ; \quad r_{i}=x_{i}(X)-x_{i}(\xi) ; \quad r_{, i}=\frac{\partial r}{\partial x_{i}(X)}=\frac{r_{i}}{r}$ 
The fundamental traction can be written as (note the derivative of the Heaviside function with respect to $\tau$ ):

$$
\begin{aligned}
2 \pi \rho c_{\mathrm{s}} & p_{i k}^{*}(X, t ; \xi, \tau) \\
= & A_{i k}\left(r L_{\mathrm{s}}^{3} H_{\mathrm{s}}+L_{\mathrm{s}} \frac{\partial}{\partial\left(c_{\mathrm{s}} \tau\right)} H_{\mathrm{s}}\right)+B_{i k} N_{\mathrm{s}} L_{\mathrm{s}} H_{\mathrm{s}} \\
& +\frac{D_{i k}}{r^{2}}\left(r^{3} L_{s}^{3} H_{s}+N_{s} L_{s} \frac{\partial}{\partial\left(c_{s} \tau\right)} H_{\mathrm{s}}\right) \\
& -\frac{c_{\mathrm{s}}}{c_{\mathrm{d}}}\left[B_{i k} N_{\mathrm{d}} L_{\mathrm{d}} H_{\mathrm{d}}+\frac{D_{i k}}{r^{2}}\left(r^{3} L_{\mathrm{d}}^{3} H_{\mathrm{d}}+N_{\mathrm{d}} L_{\mathrm{d}} \frac{\partial}{\partial\left(c_{\mathrm{d}} \tau\right)} H_{\mathrm{d}}\right)\right]
\end{aligned}
$$

The tensors $A_{i k}, B_{i k}, D_{i k}$ are given by

$A_{i k}=G\left(2 \theta n_{k} r_{, i}+\frac{\partial r}{\partial n} \delta_{i k}+n_{i} r_{, k}\right)$

$B_{i k}=-\frac{2 G}{r^{3}}\left(\frac{\partial r}{\partial n} \delta_{i k}+n_{i} r_{, k}+n_{k} r_{, i}-4 \frac{\partial r}{\partial n} r_{, i} r_{, k}\right)$

$D_{i k}=-2 G\left(\theta n_{k} r_{, i}+\frac{\partial r}{\partial n} r_{, i} r_{, k}\right)$

where:

$\theta=\frac{c_{\mathrm{d}}^{2}-2 c_{\mathrm{s}}^{2}}{2 c_{\mathrm{s}}}$

In the above expressions $c_{\mathrm{s}}$ and $c_{\mathrm{d}}$ represent the secondary (shear) and primary (dilatational) wave velocities, respectively, defined by

$c_{\mathrm{d}}^{2}=\frac{2 G}{\rho} \frac{(1-\nu)}{(1-2 \nu)}$

$c_{\mathrm{s}}^{2}=\frac{G}{\rho}$

where $\rho$ is the constant mass density, $G$ is the shear modulus and $\nu$ is the Poisson coefficient.

In expressions (8)-(10) $n$ stands for the coordinate in the direction of the outward unity vector normal to the boundary ( $n_{k}$ is its component in the $k$-direction). Additionally, in the functions below, which appear in Eqs. (2) and (7), the subscript $\mathrm{w}$ can be conveniently substituted by $\mathrm{s}$ or by $\mathrm{d}$ in order to represent contributions of secondary or primary waves, respectively:

$H_{\mathrm{w}}=H\left[c_{\mathrm{w}}(t-\tau)-r\right]$

$L_{\mathrm{w}}=L_{\mathrm{w}}(X, t ; \xi, \tau)=\left[\left(c_{\mathrm{w}}^{2}(t-\tau)^{2}-r^{2}\right]^{-1 / 2}\right.$

$N_{\mathrm{w}}=N_{\mathrm{w}}(X, t ; \xi, \tau)=2 c_{\mathrm{w}}^{2}(t-\tau)^{2}-r^{2}$

According to the treatment given to the derivatives of the Heaviside function in expression (7), two apparently different, but entirely equivalent, versions of the TD-BEM arise: the first is the version presented by Mansur [9], based on kernel regularization procedures; the second is based on the concept of finite part integrals, as described by Hadamard [12]. A complete discussion concerning these formulations (or representations) is beyond the scope of this work and can be found, for instance, in $[13,14]$. Eq. (17) below is used to describe the TD-BEM formulation employed here. One has:

$$
\begin{aligned}
& 2 \pi \rho c_{\mathrm{s}} \int_{\Gamma} \int_{0}^{t^{+}} p_{i k}^{*} u_{k} \mathrm{~d} \tau \mathrm{d} \Gamma \\
& =\int_{\Gamma}\left(A_{i k}+D_{i k}\right)\left(\int_{0}^{t^{+}} r L_{\mathrm{s}}^{3} u_{k} H_{\mathrm{s}} \mathrm{d} \tau\right) \mathrm{d} \Gamma \\
& \quad+\int_{\Gamma} B_{i k} \int_{0}^{t^{+}} L_{\mathrm{s}} N_{\mathrm{s}} u_{k} H_{\mathrm{s}} \mathrm{d} \tau \mathrm{d} \Gamma-\frac{c_{\mathrm{s}}}{c_{\mathrm{d}}} \\
& \quad \times \int_{\Gamma} B_{i k} \int_{0}^{t^{+}} L_{\mathrm{d}} N_{\mathrm{d}} u_{k} H_{\mathrm{d}} \mathrm{d} \tau \mathrm{d} \Gamma-\frac{c_{\mathrm{s}}}{c_{\mathrm{d}}} \\
& \quad \times \int_{\Gamma} D_{i k}\left(\int_{0}^{t^{+}} r L_{\mathrm{d}}^{3} u_{k} H_{\mathrm{d}} \mathrm{d} \tau\right) \mathrm{d} \Gamma
\end{aligned}
$$

The finite part integral that appears in the first and fourth time integrals on the right-hand-side of expression (17) is to be interpreted as:

$$
=\int_{0}^{t^{+}} r L_{\mathrm{w}}^{3} u_{k} H_{\mathrm{w}} \mathrm{d} \tau=\lim _{\tau \rightarrow t-r / c_{\mathrm{w}}}\left\{\int_{0}^{\tau} r L_{\mathrm{w}}^{3} u_{k} \mathrm{~d} \tau-\frac{1}{c_{\mathrm{w}}} L_{\mathrm{w}} u_{k}\right\}
$$

The description based on Hadamard finite part integral was preferred because it leads to expressions shorter than those obtained using kernel regularization.

The solution algorithm used here employs linear elements to discretize the boundary; the spatial integration is carried out numerically by Gaussian quadrature. The $C_{i k}$ coefficient on the left hand side of Eq. (1) is the same as that of the static case and can be incorporated directly in the matrix $\mathbf{H}$ related to the final time of analysis, $t_{n+1}$, (matrix $\mathbf{H}^{(n+1)(n+1)}$ in Eq. (23)) or indirectly, by adding and subtracting the static fundamental traction from the time-dependent fundamental traction. Proceeding this way, the singularities are removed and the rigid body movement technique can be used to take into account the contribution of the coefficients $C_{i k}$ together with the elements of the matrix $\mathbf{H}^{\text {static }}\left(\mathbf{H}^{\text {static }}\right.$ is assembled by employing the static fundamental traction, see expression (29) of the third section).

The aforementioned final time of analysis is given by: $t_{n+1}=(n+1) \Delta t_{\mathrm{TD}}$, where $\Delta t_{\mathrm{TD}}$ is the selected time-step for the TD-BEM sub-domain. 
By assuming linear time variation for the displacements, for a given time interval, say $\left[t_{m}, t_{m+1}\right]$, one has

$\mathbf{u}=\Phi_{\mathrm{I}}(\tau) \mathbf{u}\left(X, t_{m}\right)+\Phi_{\mathrm{F}}(\tau) \mathbf{u}\left(X, t_{m+1}\right)$

where

$\Phi_{\mathrm{I}}(\tau)=\frac{t_{m+1}-\tau}{\Delta t_{\mathrm{DT}}} ; \quad \Phi_{\mathrm{F}}(\tau)=\frac{\tau-t_{m}}{\Delta t_{\mathrm{DT}}}$

Constant time variation is assumed for the tractions; thus, one can write

$\mathbf{p}=\theta_{\mathrm{F}}(\tau) \mathbf{p}\left(X, t_{m+1}\right)$

where

$\theta_{\mathrm{F}}(\tau)=1$

Time integration is carried out, as is usual in timedomain BEM formulations, analytically. The reader is referred to $[9,10,14]$ for additional details concerning this topic. Once all the integrations have been performed, a resulting system of equations can be written, in matrix form, as follows

$$
\begin{aligned}
& \mathbf{H}^{(n+1)(n+1)} \mathbf{u}^{(n+1)} \\
& \quad=\mathbf{G}^{(n+1)(n+1)} \mathbf{p}^{(n+1)}+\sum_{m=0}^{n}\left(\mathbf{G}^{(n m)} \mathbf{p}^{(m)}-\mathbf{H}^{(n m)} \mathbf{u}^{(m)}\right)
\end{aligned}
$$

After the introduction of the boundary conditions, the following system of equations is obtained:

$\mathbf{A y}{ }^{(n+1)}=\mathbf{f}^{(n+1)}+\sum_{m=0}^{n} \mathbf{h}^{(m)}$

It is important to mention the role that the time translation property performs during the assemblage of matrices $\mathbf{H}^{(n m)}$ and $\mathbf{G}^{(n m)}, m=0,1,2, \ldots, n ; n=1,2,3, \ldots$, avoiding repetitive and unnecessary operations that could, for large values of $t_{\mathrm{n}+1}$, make the analysis very expensive (from the computational point of view). The time translation property states that:

$u_{i j}^{*}(x, t, \xi, \tau)=u_{i j}^{*}\left(x, t+t_{1}, \xi, \tau+t_{1}\right)$

Vector $\mathbf{y}^{(n+1)}$ in Eq. (24) contains the unknown values of displacements and tractions, whereas the boundary conditions contributions at the current time are stored in vector $\mathbf{f}^{(n+1)}$. Previous time history contribution, represented by the convolution, is included in vectors $\mathbf{h}^{(m)}$.

It is important to mention that the convolution can be computed in a more economic way; in other words, not all the matrices indicated by Eq. (23) need to be evaluated. The truncation scheme proposed by Soares Jr. and Mansur [11] reduces substantially the CPU time required to perform the time integration with no accuracy loss.

An adequate choice of the time-step plays a fundamental role in the analysis. The parameter $\beta_{\mathrm{TD}}$ defined by Mansur [9] was used to estimate the time-step duration. For the TDBEM formulation, reference will be made to $\beta_{\mathrm{TD}}$ given by
$\beta_{\mathrm{TD}}=\frac{c_{\mathrm{d}} \Delta t_{\mathrm{TD}}}{l}$

where $l$ is the smallest boundary element length of the TDBEM sub-domain.

A good estimative of the value of the parameter $\beta_{\mathrm{TD}}$ requires experience with the method. Diversely from the FEM formulation, where the definition of the critical timestep can be done appropriately $[15,16]$, in BEM formulations, as can be observed, for instance, in the works by Mansur [9], Carrer and Mansur [14], Partridge et al. [4], Kontoni and Beskos [3] and Hatzigeorgiou and Beskos [17], there is no rigorous mathematical study concerning this matter. For this reason, experience plays a fundamental role in the choice of the time-step. The simplest and straightforward recommendation is to avoid values of $\beta_{\mathrm{TD}}$ either much greater than unity or too small. In the TD-BEM formulation, values of $\beta_{\mathrm{TD}}$ equal to 0.6 are recommended.

\section{D-BEM formulation}

The D-BEM formulation requires discretization of the entire domain in order to take into account the influence of the inertial terms. Although at a first glance this feature may seem disappointing, the simplicity and universality of this formulation, allied to the good results it can provide, fully justify its use in this area of research.

As mentioned earlier, in the coupling procedure presented in this paper, the D-BEM formulation is employed for sub-domains where non-linear material behaviour is expected to occur, see [1] for a description concerning the BEM elastoplastic formulation. The starting D-BEM integral equation, associated with the initial stress formulation is written as follows (note the presence of two domain integrals: one related to the acceleration, ü, and another one related to the initial stress, $\sigma^{\mathrm{p}}$ ), as described by Carrer and Telles [2]:

$$
\begin{aligned}
& C_{i k}(\xi) u_{k}(\xi, t) \\
& =\int_{\Gamma} u_{i k}^{*}(\xi, X) p_{k}(X, t) \mathrm{d} \Gamma(X)-\int_{\Gamma} p_{i k}^{*}(\xi, X) u_{k}(X, t) \mathrm{d} \Gamma(X) \\
& \quad-\rho \int_{\Omega} u_{i k}^{*}(\xi, X) \mathrm{u}_{k}(X, t) \mathrm{d} \Omega(X)+\int_{\Omega} \varepsilon_{k l i}^{*}(\xi, X) \sigma_{k l}^{\mathrm{p}}(X, t) \mathrm{d} \Omega(X)
\end{aligned}
$$

For plane strain problems, one has

$u_{i k}^{*}(\xi, X)=\frac{1}{8 \pi G(1-\nu)}\left[(3-4 \nu) \ln (1 / r) \delta_{i k}+r_{, i} r_{, k}\right]$

and 


$$
\begin{aligned}
p_{i k}^{*}(\xi, X) & =-\frac{1}{4 \pi(1-\nu) r} \\
& {\left[\frac{\partial r}{\partial n}\left((1-2 \nu) \delta_{i k}+2 r_{, i} r_{, k}\right)-(1-2 \nu)\left(r_{, i} n_{k}-r_{, k} n_{i}\right)\right] }
\end{aligned}
$$

Plane strain expressions are valid for plane stress if $\nu$ is replaced by $\bar{\nu}=\nu /(1+\nu)$.

Stress components, for internal points $\left(C_{i k}=\delta_{i k}\right)$, can be computed according to:

$$
\begin{aligned}
\sigma_{i k}(\xi ; t)= & \int_{\Gamma} u_{i k j}^{*}(\xi, X) p_{j}(X, t) \mathrm{d} \Gamma(X) \\
& -\int_{\Gamma} p_{i k j}^{*}(\xi, X) u_{j}(X, t) \mathrm{d} \Gamma(X) \\
& -\rho \int_{\Omega} u_{i k j}^{*}(\xi, X) \mathrm{u}_{j}(X, t) \mathrm{d} \Omega(X) \\
& +\int_{\Omega} \varepsilon_{i k j l}^{*}(\xi, X) \sigma_{j l}^{p}(X, t) \mathrm{d} \Omega(X)+g_{i k}\left[\sigma_{j l}^{\mathrm{p}}(\xi ; t)\right]
\end{aligned}
$$

It is important to note that the initial stress domain integral, in Eq. (30), must be evaluated in the Cauchy principal value sense and that the so-called free term $g_{i k}$ appears, according to Telles [1], due to the derivative of the domain integral related to the initial stress shown in Eq. (27). The expressions for computing the function $\varepsilon_{k l i}^{*}(\xi, X)$ (see Eq. (27)), the function $\varepsilon_{i k j l}^{*}(\xi, X)$ and the free term $g_{i k}[$ $\left.\sigma_{j l}^{\mathrm{p}}(\xi, t)\right]$ (see Eq. (30)) can be found [1].

The acceleration that appears in Eq. (27) is approximated by recurrence formulae involving displacements at different time levels. As a consequence, the unknowns to be determined at a given time, say $t_{n+1}$, are the displacements and the tractions on the boundary, and the displacements at the internal points. In this way, not only the part of the domain where inelastic terms are expected to occur must be discretized, as is done in static analyses; now, due to the presence of the inertial term, the entire domain has to be discretized. Here, linear triangular cells are employed in this discretization.

The application of the discretized version of Eq. (27) to all boundary nodes and internal points produces an enlarged system of equations, schematically written as [2]:

$$
\begin{aligned}
& {\left[\begin{array}{ll}
\mathbf{H}^{\mathrm{bb}} & \mathbf{0} \\
\mathbf{H}^{\mathrm{db}} & \mathbf{I}
\end{array}\right]\left\{\begin{array}{l}
\mathbf{u}^{\mathrm{b},(n+1)} \\
\mathbf{u}^{\mathrm{d},(n+1)}
\end{array}\right\}=\left[\begin{array}{l}
\mathbf{G}^{\mathrm{bb}} \\
\mathbf{G}^{\mathrm{db}}
\end{array}\right]\left\{\mathbf{p}^{\mathrm{b},(n+1)}\right\}} \\
& -\left[\begin{array}{ll}
\mathbf{M}^{\mathrm{bb}} & \mathbf{M}^{\mathrm{bd}} \\
\mathbf{M}^{\mathrm{db}} & \mathbf{M}^{\mathrm{dd}}
\end{array}\right]\left\{\begin{array}{c}
\mathbf{u}^{\mathrm{b},(n+1)} \\
\mathbf{u}^{\mathrm{d},(n+1)}
\end{array}\right\} \\
& +\left[\begin{array}{ll}
\mathbf{Q}^{\mathrm{bb}} & \mathbf{Q}^{\mathrm{bd}} \\
\mathbf{Q}^{\mathrm{db}} & \mathbf{Q}^{\mathrm{dd}}
\end{array}\right]\left\{\begin{array}{c}
\boldsymbol{\sigma}^{\mathrm{p}, \mathrm{b},(n+1)} \\
\boldsymbol{\sigma}^{\mathrm{p}, \mathrm{d},(n+1)}
\end{array}\right\}
\end{aligned}
$$

In Eq. (31) the superscripts $b$ and d correspond to the boundary and to the domain (internal points), respectively. In the sub-matrices, the first superscript corresponds to the position of the source point and the second superscript corresponds to the position of the field point. The identity matrix I is related to the coefficients $C_{i k}=\delta_{i k}$ of the internal points.

The simultaneous evaluation of the stress components at boundary nodes and internal points, by its turn, is accomplished by the system of equations below:

$$
\begin{aligned}
& \left\{\begin{array}{l}
\boldsymbol{\sigma}^{\mathrm{b},(n+1)} \\
\boldsymbol{\sigma}^{\mathrm{d},(n+1)}
\end{array}\right\}=\left[\begin{array}{l}
\mathbf{G}^{\prime \mathrm{bb}} \\
\mathbf{G}^{\prime \mathrm{db}}
\end{array}\right]\left\{\mathbf{p}^{\mathrm{b},(n+1)}\right\}-\left[\begin{array}{l}
\mathbf{H}^{\mathrm{\prime bb}} \\
\mathbf{H}^{\prime \mathrm{db}}
\end{array}\right]\left\{\mathbf{u}^{\mathrm{b},(n+1)}\right\} \\
& -\left[\begin{array}{cc}
\mathbf{0} \\
\mathbf{M}^{\prime \mathrm{db}} \mathbf{M}^{\prime \mathrm{dd}}
\end{array}\right]\left\{\begin{array}{l}
\mathbf{u}^{\mathrm{b},(n+1)} \\
\mathbf{u}^{\mathrm{d},(n+1)}
\end{array}\right\} \\
& +\left[\begin{array}{l}
\mathbf{Q}^{\mathrm{\prime bb}} \mathbf{0} \\
\mathbf{Q}^{\prime \mathrm{dbb}}\left(\mathbf{Q}^{\prime \mathrm{dd}}+\mathbf{E}^{\prime}\right)
\end{array}\right]\left\{\begin{array}{l}
\boldsymbol{\sigma}^{\mathrm{p}, \mathrm{b},(n+1)} \\
\boldsymbol{\sigma}^{\mathrm{p}, \mathrm{d},(n+1)}
\end{array}\right\}
\end{aligned}
$$

Note that the contribution of the free term $g_{i k}$ is included in matrix $\mathbf{E}^{\prime}$.

\subsection{Time-marching scheme}

For the D-BEM approach, Houbolt scheme was adopted to march on time. Apparently, the artificial damping, noticeable when FEM analyses are carried out with the Houbolt scheme [15] are beneficial in BEM analyses, turning this scheme the most appropriate one for D-BEM analyses. It is important to point out that the use of the Newmark scheme [18] on the other hand, tends to produce amplification of the numerical results, leading to unreliable responses. The alternative schemes proposed by Souza et al. [7] and Carrer and Mansur [6] intend to provoke a discussion on this matter and could, as well, be implemented; however, these schemes were not implemented, as a discussion concerning time integration is beyond the scope of the present article.

In the Houbolt scheme, the following approximations are adopted, respectively, for the velocity and acceleration [5]

$$
\begin{aligned}
\dot{\mathbf{u}}^{(n+1)}= & \frac{1}{6 \Delta t}\left[11 \mathbf{u}^{(n+1)}-18 \mathbf{u}^{(n)}+9 \mathbf{u}^{(n-1)}\right. \\
& \left.-2 \mathbf{u}^{(n-2)}\right]
\end{aligned}
$$

and

$\mathbf{u}^{(n+1)}=\frac{1}{\Delta t^{2}}\left[2 \mathbf{u}^{(n+1)}-5 \mathbf{u}^{(n)}+4 \mathbf{u}^{(n-1)}-\mathbf{u}^{(n-2)}\right]$

The substitution of expression (34) into expressions (31) and (32) gives 


$$
\begin{aligned}
& {\left[\begin{array}{ll}
\mathbf{H}^{\prime \mathrm{bb}} & \mathbf{0} \\
\mathbf{H}^{\prime \mathrm{db}} & \mathbf{I}
\end{array}\right]\left\{\begin{array}{l}
\mathbf{u}^{\mathrm{b},(n+1)} \\
\mathbf{u}^{\mathrm{d},(n+1)}
\end{array}\right\}} \\
& =\left[\begin{array}{l}
\mathbf{G}^{\mathrm{bb}} \\
\mathbf{G}^{\mathrm{db}}
\end{array}\right]\left\{\mathbf{p}^{\mathrm{b},(n+1)}\right\}-\left[\begin{array}{ll}
\mathbf{M}^{\mathrm{bb}} & \mathbf{M}^{\mathrm{bd}} \\
\mathbf{M}^{\mathrm{db}} & \mathbf{M}^{\mathrm{dd}}
\end{array}\right] \\
& \times \frac{1}{\Delta t^{2}}\left\{\begin{array}{l}
2 \mathbf{u}^{\mathrm{b},(n+1)}-5 \mathbf{u}^{\mathrm{b},(n)}+4 \mathbf{u}^{\mathrm{b},(n-1)}-\mathbf{u}^{\mathrm{b},(n-2)} \\
2 \mathbf{u}^{\mathrm{d},(n+1)}-5 \mathbf{u}^{\mathrm{d},(n)}+4 \mathbf{u}^{\mathrm{d},(n-1)}-\mathbf{u}^{\mathrm{d},(n-2)}
\end{array}\right\} \\
& +\left[\begin{array}{ll}
\mathbf{Q}^{\mathrm{bb}} & \mathbf{Q}^{\mathrm{bd}} \\
\mathbf{Q}^{\mathrm{db}} & \mathbf{Q}^{\mathrm{dd}}
\end{array}\right]\left\{\begin{array}{l}
\boldsymbol{\sigma}^{\mathrm{p}, \mathrm{b},(n+1)} \\
\boldsymbol{\sigma}^{\mathrm{p}, \mathrm{d},(n+1)}
\end{array}\right\}
\end{aligned}
$$

and

$$
\begin{aligned}
&\left\{\begin{array}{l}
\boldsymbol{\sigma}^{b,(n+1)} \\
\boldsymbol{\sigma}^{d,(n+1)}
\end{array}\right\}= {\left[\begin{array}{l}
\mathbf{G}^{\prime \mathrm{bb}} \\
\mathbf{G}^{\prime \mathrm{db}}
\end{array}\right]\left\{\mathbf{p}^{b,(n+1)}\right\} } \\
&-\left[\begin{array}{l}
\mathbf{H}^{\prime \mathrm{bb}} \\
\mathbf{H}^{\prime \mathrm{db}}
\end{array}\right]\left\{\mathbf{u}^{b,(n+1)}\right\} \\
&-\left[\begin{array}{ll}
\mathbf{0} & \mathbf{0} \\
\mathbf{M}^{\prime \mathrm{db}} & \mathbf{M}^{\prime \mathrm{dd}}
\end{array}\right] \\
& \times \frac{1}{\Delta t^{2}}\left\{\begin{array}{l}
2 \mathbf{u}^{b,(n+1)}-5 \mathbf{u}^{b,(n)}+4 \mathbf{u}^{b,(n-1)}-\mathbf{u}^{b,(n-2)} \\
2 \mathbf{u}^{d,(n+1)}-5 \mathbf{u}^{d,(n)}+4 \mathbf{u}^{d,(n-1)}-\mathbf{u}^{d,(n-2)}
\end{array}\right\} \\
&+\left[\begin{array}{ll}
\mathbf{Q}^{\prime \mathrm{bb}} & \mathbf{0} \\
\mathbf{Q}^{\prime \mathrm{db}} & \mathbf{Q}^{\prime \mathrm{dd}}+\mathbf{E}^{\prime}
\end{array}\right]\left\{\begin{array}{l}
\boldsymbol{\sigma}^{p, b,(n+1)} \\
\boldsymbol{\sigma}^{p, b,(n+1)}
\end{array}\right\}
\end{aligned}
$$

After rearranging common terms in Eqs. (35) and (36), one has

$$
\begin{aligned}
& {\left[\begin{array}{ll}
\left(\Delta t^{2} \mathbf{H}^{\mathrm{bb}}+2 \mathbf{M}^{\mathrm{bb}}\right) & \left(\mathbf{0}+2 \mathbf{M}^{\mathrm{bd}}\right) \\
\left(\Delta t^{2} \mathbf{H}^{\mathrm{db}}+2 \mathbf{M}^{\mathrm{db}}\right) & \left(\Delta t^{2} \mathbf{I}+2 \mathbf{M}^{\mathrm{dd}}\right)
\end{array}\right]} \\
& \left\{\begin{array}{c}
\mathbf{u}^{b,(n+1)} \\
\mathbf{u}^{d,(n+1)}
\end{array}\right\}=\Delta t^{2}\left[\begin{array}{l}
\mathbf{G}^{\mathrm{bb}} \\
\mathbf{G}^{\mathrm{db}}
\end{array}\right]\left\{\mathbf{p}^{b,(n+1)}\right\}-\left[\begin{array}{ll}
\mathbf{M}^{\mathrm{bb}} & \mathbf{M}^{\mathrm{bd}} \\
\mathbf{M}^{\mathrm{db}} & \mathbf{M}^{\mathrm{dd}}
\end{array}\right] \\
& \left\{\begin{array}{c}
-5 \mathbf{u}^{b,(n)}+4 \mathbf{u}^{b,(n-1)}-\mathbf{u}^{b,(n-2)} \\
-5 \mathbf{u}^{d,(n)}+4 \mathbf{u}^{d,(n-1)}-\mathbf{u}^{d,(n-2)}
\end{array}\right\} \\
& +\Delta t^{2}\left[\begin{array}{ll}
\mathbf{Q}^{\mathrm{bb}} & \mathbf{Q}^{\mathrm{bd}} \\
\mathbf{Q}^{\mathrm{db}} & \mathbf{Q}^{\mathrm{dd}}
\end{array}\right]\left\{\begin{array}{c}
\boldsymbol{\sigma}^{p, b,(n+1)} \\
\boldsymbol{\sigma}^{p, d,(n+1)}
\end{array}\right\}
\end{aligned}
$$

and

$$
\begin{aligned}
& \left\{\begin{array}{l}
\boldsymbol{\sigma}^{b,(n+1)} \\
\boldsymbol{\sigma}^{d,(n+1)}
\end{array}\right\}=\left[\begin{array}{l}
\mathbf{G}^{\prime \mathrm{bb}} \\
\mathbf{G}^{\prime \mathrm{db}}
\end{array}\right]\left\{\mathbf{p}^{b,(n+1)}\right\} \\
& -\left[\begin{array}{l}
\mathbf{H}^{\prime \mathrm{bb}} \\
\mathbf{H}^{\prime \mathrm{db}}+\frac{2}{\Delta t^{2}} \mathbf{M}^{\prime \mathrm{db}}
\end{array}\right]\left\{\mathbf{u}^{b,(n+1)}\right\} \\
& -\left[\begin{array}{ll}
\mathbf{0} & \mathbf{0} \\
\mathbf{M}^{\prime \mathrm{db}} & \mathbf{M}^{\prime \mathrm{dd}}
\end{array}\right] \frac{1}{\Delta t^{2}} \\
& \left\{\begin{array}{l}
-5 \mathbf{u}^{b,(n)}+4 \mathbf{u}^{b,(n-1)}-\mathbf{u}^{b,(n+2)} \\
2 \mathbf{u}^{d,(n+1)}-5 \mathbf{u}^{d,(n-1)}+4 \mathbf{u}^{d,(n-1)}-\mathbf{u}^{d,(n-2)}
\end{array}\right\} \\
& +\left[\begin{array}{ll}
\mathbf{Q}^{\prime \mathrm{bb}} & \mathbf{0} \\
\mathbf{Q}^{\prime \mathrm{db}} & \left(\mathbf{Q}^{\prime d \mathrm{~d}}+\mathbf{E}^{\prime}\right)
\end{array}\right]\left\{\begin{array}{l}
\boldsymbol{\sigma}^{p, b,(n+1)} \\
\boldsymbol{\sigma}^{p, d,(n+1)}
\end{array}\right\}
\end{aligned}
$$

After the boundary conditions are imposed, the system of Eqs. (37) and (38) can be written as

$$
\begin{aligned}
& {\left[\begin{array}{ll}
\mathbf{A}^{\mathrm{bb}} & \left(\mathbf{0}+\mathbf{M}^{\mathrm{bd}}\right) \\
\mathbf{A}^{\mathrm{db}} & \left(\Delta t^{2} \mathbf{I}+2 \mathbf{M}^{\mathrm{dd}}\right)
\end{array}\right]\left\{\begin{array}{l}
\mathbf{y}^{b,(n+1)} \\
\mathbf{u}^{d,(n+1)}
\end{array}\right\}} \\
& =\left[\begin{array}{l}
\mathbf{B}^{\mathrm{bb}} \\
\mathbf{B}^{\mathrm{db}}
\end{array}\right]\left\{\mathbf{b}^{b,(n+1)}\right\} \\
& -\left[\begin{array}{ll}
\mathbf{M}^{\mathrm{bb}} & \mathbf{M}^{\mathrm{bd}} \\
\mathbf{M}^{\mathrm{db}} & \mathbf{M}^{\mathrm{dd}}
\end{array}\right]\left\{\begin{array}{l}
-5 \mathbf{u}^{b,(n)}+4 \mathbf{u}^{b,(n-1)}-\mathbf{u}^{b,(n-1)} \\
-5 \mathbf{u}^{d,(n)}+4 \mathbf{u}^{d,(n-1)}-\mathbf{u}^{d,(n-1)}
\end{array}\right\} \\
& +\Delta t^{2}\left[\begin{array}{ll}
\mathbf{Q}^{\mathrm{bb}} & \mathbf{Q}^{\mathrm{bd}} \\
\mathbf{Q}^{\mathrm{db}} & \mathbf{Q}^{\mathrm{dd}}
\end{array}\right]\left\{\begin{array}{l}
\boldsymbol{\sigma}^{p, b,(n+1)} \\
\boldsymbol{\sigma}^{p, d,(n+1)}
\end{array}\right\}
\end{aligned}
$$

and

$$
\begin{aligned}
& \left\{\begin{array}{l}
\boldsymbol{\sigma}^{b,(n+1)} \\
\boldsymbol{\sigma}^{d,(n+1)}
\end{array}\right\}=-\left[\begin{array}{ll}
\mathbf{A}^{\prime \mathrm{bb}} & \mathbf{0} \\
\mathbf{A}^{\prime \mathrm{db}} & +\frac{2}{\Delta t^{2}} \mathbf{M}^{\prime \mathrm{dd}}
\end{array}\right]\left\{\begin{array}{l}
\mathbf{y}^{b,(n+1)} \\
\mathbf{u}^{d,(n+1)}
\end{array}\right\} \\
& +\left[\begin{array}{l}
\mathbf{B}^{\mathrm{bb}} \\
\mathbf{B}^{/ \mathrm{db}}
\end{array}\right]\left\{\mathbf{b}^{b,(n+1)}\right\}-\left[\begin{array}{ll}
\mathbf{0} & \mathbf{0} \\
\mathbf{M}^{\prime \mathrm{db}} & \mathbf{M}^{\prime \mathrm{dd}}
\end{array}\right] \\
& \times \frac{1}{\Delta t^{2}}\left\{\begin{array}{l}
-5 \mathbf{u}^{b,(n)}+4 \mathbf{u}^{b,(n-1)}-\mathbf{u}^{b,(n-2)} \\
-5 \mathbf{u}^{d,(n)}+4 \mathbf{u}^{d,(n-1)}-\mathbf{u}^{d,(n-2)}
\end{array}\right\} \\
& +\left[\begin{array}{ll}
\mathbf{Q}^{\prime \mathrm{bb}} & \mathbf{0} \\
\mathbf{Q}^{\prime \mathrm{db}} & \left(\mathbf{Q}^{\prime \mathrm{dd}}+\mathbf{E}^{\prime}\right)
\end{array}\right]\left\{\begin{array}{l}
\boldsymbol{\sigma}^{p, b,(n+1)} \\
\boldsymbol{\sigma}^{p, d,(n+1)}
\end{array}\right\}
\end{aligned}
$$

Matrices $\mathbf{A}^{\mathrm{bb}}$ and $\mathbf{A}^{\mathrm{db}}$, in Eq. (39), are constituted by columns of matrices $\mathbf{H}^{\mathrm{bb}}$ and $\mathbf{H}^{\mathrm{db}}$ (for unknown 
displacements) or by columns of matrices $\mathbf{G}^{\mathrm{bb}}$ and $\mathbf{G}^{\mathrm{db}}$ (for unknown tractions). Matrices $\mathbf{B}^{\mathrm{bb}}$ and $\mathbf{B}^{\mathrm{db}}$ are constituted in a similar way, but are associated with the known boundary prescribed values already included in vector $\mathbf{b}^{n+1}$. The same interpretation is valid for Eq. (40).

In order to provide a simpler visualization of the matrix operations carried out, when necessary a compact notation will be employed. Bearing this remark in mind, Eq. (39) can be written as:

$$
\begin{aligned}
\mathbf{A y}{ }^{(n+1)}= & \mathbf{B} \mathbf{b}^{(n+1)}-\mathbf{M}\left(-5 \mathbf{u}^{(n)}+4 \mathbf{u}^{(n-1)}-\mathbf{u}^{(n-2)}\right) \\
& +\mathbf{Q} \boldsymbol{\sigma}^{p,(n+1)}
\end{aligned}
$$

Finally, one can write

$$
\begin{aligned}
\left\{\begin{array}{l}
\mathbf{y}^{b,(n+1)} \\
\mathbf{u}^{d,(n+1)}
\end{array}\right\}= & \left\{\begin{array}{l}
\mathbf{m}^{b,(n+1)} \\
\mathbf{m}^{d,(n+1)}
\end{array}\right\} \\
& +\left[\begin{array}{ll}
\mathbf{R}^{\mathrm{bb}} & \mathbf{R}^{\mathrm{bd}} \\
\mathbf{R}^{\mathrm{db}} & \mathbf{R}^{\mathrm{dd}}
\end{array}\right]\left\{\begin{array}{l}
\boldsymbol{\sigma}^{p, b(n+1)} \\
\boldsymbol{\sigma}^{p, d,(n+1)}
\end{array}\right\}
\end{aligned}
$$

or

$\mathbf{y}^{(n+1)}=\mathbf{m}^{(n+1)}+\mathbf{R} \boldsymbol{\sigma}^{p,(n+1)}$

In the above expression, matrix $\mathbf{R}$ is given by

$$
\mathbf{R}=\mathbf{A}^{-1} \mathbf{Q}
$$

and $\mathbf{m}^{n+1}$ corresponds to the elastic response (boundary and internal points displacements and boundary tractions), computed according to:

$\mathbf{m}^{(n+1)}=A^{-1}\left[\mathbf{B b}^{(n+1)}-\mathbf{M}\left(-5 \mathbf{u}^{(n)}+4 \mathbf{u}^{(n-1)}-\mathbf{u}^{(n-2)}\right)\right]$

Substituting Eq. (42) into Eq. (40) and grouping common terms one has the following system of equations related to the stress computation:

$$
\begin{aligned}
& \left\{\begin{array}{l}
\boldsymbol{\sigma}^{b,(n+1)} \\
\boldsymbol{\sigma}^{d,(n+1)}
\end{array}\right\}=-\left[\begin{array}{ll}
\mathbf{A}^{\prime b b} & \mathbf{0} \\
\mathbf{A}^{\prime d \mathrm{~b}} & +\frac{2}{\Delta t^{2}} \mathbf{M}^{\prime d d}
\end{array}\right]\left\{\begin{array}{l}
\mathbf{m}^{b,(n+1)} \\
\mathbf{m}^{d,(n+1)}
\end{array}\right\} \\
& +\left[\begin{array}{l}
\mathbf{B}^{\prime \mathrm{bb}} \\
\mathbf{B}^{\prime \mathrm{db}}
\end{array}\right]\left\{\mathbf{b}^{b,(n+1)}\right\}-\left[\begin{array}{ll}
\mathbf{0} & \mathbf{0} \\
\mathbf{M}^{\prime \mathrm{db}} & \mathbf{M}^{\prime \mathrm{dd}}
\end{array}\right] \\
& \times \frac{1}{\Delta t^{2}}\left\{\begin{array}{c}
-5 \mathbf{u}^{b,(n)}+4 \mathbf{u}^{b,(n-1)}-\mathbf{u}^{b,(n-2)} \\
-5 \mathbf{u}^{d,(n)}+4 \mathbf{u}^{d,(n-1)}-\mathbf{u}^{b,(n-2)}
\end{array}\right\} \\
& \begin{array}{l}
+\left[-\left[\begin{array}{ll}
\mathbf{A}^{\prime \mathrm{bb}} & \mathbf{0} \\
\mathbf{A}^{\prime \mathrm{db}} & +\frac{2}{\Delta t^{2}} \mathbf{M}^{\prime \mathrm{dd}}
\end{array}\right]\left[\begin{array}{ll}
\mathbf{R}^{\mathrm{bb}} & \mathbf{R}^{\mathrm{bd}} \\
\mathbf{R}^{\mathrm{db}} & \mathbf{R}^{\mathrm{dd}}
\end{array}\right.\right. \\
\left.+\left[\begin{array}{ll}
\mathbf{Q}^{\prime \mathrm{bb}} & \mathbf{0} \\
\mathbf{Q}^{\prime \mathrm{db}} & \left(\mathbf{Q}^{\prime \mathrm{dd}}+\mathbf{E}^{\prime}\right)
\end{array}\right]\right\}\left\{\begin{array}{l}
\boldsymbol{\sigma}^{p, b,(n+1)} \\
\boldsymbol{\sigma}^{p, d,(n+1)}
\end{array}\right\}
\end{array}
\end{aligned}
$$

In compact notation

$$
\boldsymbol{\sigma}^{(n+1)}=\mathbf{n}^{(n+1)}+\mathbf{S} \boldsymbol{\sigma}^{p,(n+1)}
$$

where $\mathbf{n}^{n+1}$ represents the elastic stresses, given by

$$
\begin{aligned}
\mathbf{n}^{(n+1)}= & -\mathbf{A}^{\prime} \mathbf{m}^{(n+1)}+\mathbf{B}^{\prime} \mathbf{b}^{(n+1)}-\mathbf{M}^{\prime}\left(-5 \mathbf{u}^{(n)}\right. \\
& \left.+4 \mathbf{u}^{(n-1)}-\mathbf{u}^{(n-2)}\right)
\end{aligned}
$$

and

$\mathbf{S}=-\mathbf{A}^{\prime} \mathbf{R}+\left(\mathbf{Q}^{\prime}+\mathbf{E}^{\prime}\right)$

In the D-BEM formulation, reference will be made to the dimensionless parameter $\beta_{\mathrm{D}}$, defined similarly to the corresponding parameter of the TD-BEM formulation (Eq. (26)). Now, according to Ref. [2], the best choice is $\beta_{\mathrm{D}}=1 /$ 3.

\subsection{The non-linear problem: constitutive equations}

The solution of the non-linear problem is based on the initial stress approach. Before presenting the recursive matrix relations that characterize this form of solution, it is important to present some basic relations between the variables involved in the analysis. A brief summary is presented now.

The incremental stress-strain relations can be written as follows

$\mathrm{d} \sigma_{i j}=C_{i j k l}^{\mathrm{ep}} \mathrm{d} \varepsilon_{k l}$

where $\mathrm{d} \varepsilon_{k l}$ is the total strain increment and (in the context of associated isotropic work hardening theory)

$C_{i j k l}^{\mathrm{ep}}=C_{i j k l}-\frac{1}{\gamma^{\prime}} C_{i j m n} a_{m n} a_{o p} C_{o p k l}$

where: 
$C_{i j k l}=\frac{2 G \nu}{1-2 \nu} \delta_{i j} \delta_{k l}+G\left(\delta_{i k} \delta_{j l}+\delta_{i l} \delta_{j k}\right)$

$a_{k l}=\frac{\partial F}{\partial \sigma_{k l}}=\frac{\partial \sigma_{\mathrm{e}}}{\partial \sigma_{k l}}$

$\gamma^{\prime}=a_{i j} C_{i j k l} a_{k j}+H^{\prime}=a_{i j} d_{i j}+H^{\prime}$

$H^{\prime}=\frac{\mathrm{d} Y}{\mathrm{~d} \varepsilon_{\mathrm{e}}^{\mathrm{p}}}$

The function $Y$ plays the role of the yield stress and $\mathrm{d} \varepsilon_{\mathrm{e}}^{\mathrm{p}}$ is the increment of the equivalent or effective plastic strain.

For the implementation of the initial stress formulation, Eq. (46) (or Eq. (47) in a compact notation) can be conveniently modified with the introduction of a fictitious elastic stress tensor, defined by:

$\mathrm{d} \sigma_{i j}^{\mathrm{e}}=C_{i j k l} \mathrm{~d} \varepsilon_{k l}$

Eq. (50), thus, can be written as

$\mathrm{d} \sigma_{i j}=\mathrm{d} \sigma_{i j}^{\mathrm{e}}-\frac{1}{\gamma^{\prime}} C_{i j m n} a_{m n} a_{k l} \mathrm{~d} \sigma_{k l}^{\mathrm{e}}$

which means that the true stress tensor can be computed from the corresponding elastic stress tensor in incremental form. The increments of the initial stress tensor can be computed according to:

$\mathrm{d} \sigma_{i j}^{\mathrm{p}}=\mathrm{d} \sigma_{i j}^{\mathrm{e}}-\mathrm{d} \sigma_{i j}=\frac{1}{\gamma^{\prime}} C_{i j m n} a_{m n} a_{k l} \mathrm{~d} \sigma_{k l}^{\mathrm{e}}$

If a similar procedure is adopted in the BEM equations, it follows immediately that the increments of the elastic stress tensor can be computed directly from Eq. (30) if the free coefficient $g_{i j}$ is substituted by $\bar{g}_{i j}$, given by:

$\bar{g}_{i j}\left(\sigma_{k l}^{\mathrm{p}}\right)=g_{i j}\left(\sigma_{k l}^{\mathrm{p}}\right)+\sigma_{i j}^{\mathrm{p}}$

For numerical purposes, the increments of the elastic stress tensor can be computed directly from Eq. (46) if matrix $\mathbf{E}^{\prime}$ is replaced by $\overline{\mathbf{E}}$ :

$\overline{\mathbf{E}}=\mathbf{E}^{\prime}+\mathbf{I}$

In incremental form, one has

$\Delta \sigma^{\mathrm{e}}(t+\Delta t)=\overline{\mathbf{S}} \Delta \sigma^{\mathrm{p}}+\Delta \mathbf{n}(t+\Delta t)$

where

$\overline{\mathbf{S}}=\left(\mathbf{Q}^{\prime}+\overline{\mathbf{E}}\right)-\mathbf{A}^{\prime} \mathbf{R}$

and

$\Delta \mathbf{n}(t+\Delta t)=\mathbf{n}(t+\Delta t)-\mathbf{n}(t)$

Before presenting the solution techniques for the nonlinear problem, it is important to mention that stresses at internal points, not only in the D-BEM sub-domain but also at the TD-BEM sub-domain (if necessary), can be computed in a very accurate and simple way by adopting the method based on complex algebra described by Lyness and Moler [19]. Within the BEM context, this complex variable technique was already applied by Gao et al. [20] for static non-linear problems and by Soares Jr. et al. [21] for elastodynamic problems.

\subsection{Solution techniques}

Static and transient dynamic elastoplastic problems have been successfully solved using implicit techniques [2]. The techniques employed in this paper will be commented briefly.

The following matrix equation is obtained after Eq. (58) is applied to all boundary nodes and internal points:

$\Delta \sigma^{\mathrm{p}}=\mathbf{D}^{\mathrm{p}} \Delta \boldsymbol{\sigma}^{\mathrm{e}}$

At a fixed time, say $t_{n+1}$, the substitution of Eq. (64) in the accumulated form of Eq. (56) allows for the application of an iterative algorithm for the solution of the elastoplastic problem. The step $k$ of this algorithm can be defined according to:

${ }^{(k-1)} \boldsymbol{\sigma}^{\mathrm{e}}+{ }^{(k)} \Delta \boldsymbol{\sigma}^{\mathrm{e}}=\overline{\mathbf{S}}\left[{ }^{(k-1)} \boldsymbol{\sigma}^{\mathrm{p}}+{ }^{(k)} \mathbf{D}^{\mathrm{p}(k)} \Delta \boldsymbol{\sigma}^{\mathrm{e}}\right]+\mathbf{n}^{(n)}+\Delta \mathbf{n}^{(n+1)}$

When the residual vector defined by Eq. (66) below

$\mathrm{p}$
$\psi^{(n)}=\overline{\mathbf{S}} \boldsymbol{\sigma}^{(n)}+\mathbf{n}^{(n)}-\boldsymbol{\sigma}^{(n)}$

is substituted into expression (65), one has

$\mathbf{S}^{\mathrm{p}} \Delta \boldsymbol{\sigma}^{e,(n+1)}=\Delta \mathbf{n}^{(n+1)}+\psi^{(n)}$

where the non-linear matrix $\mathbf{S}^{\mathrm{p}}$ is given by:

$\mathbf{S}^{\mathrm{p}}=\mathbf{I}-\overline{\mathbf{S}} \mathbf{D}^{\mathrm{p}}$

Eq. (67) can be solved for each time-step. In principle, no iterations are required if sufficiently small time-steps are adopted. In practice, however, a few iterations must be performed, in order to avoid a quick deterioration of the accuracy, giving rise to the following schemes:

(i) Pure Incremental (PI). Eq. (67) is applied only once at each time-step, without iterations. Matrix $\mathbf{S}^{\mathrm{p}}$ is always updated as a function of the previous solution.

(ii) Newton-Raphson. By definition, matrix $\mathbf{S}^{\mathrm{p}}$ is updated after each iteration. For the first iteration of a given time-step, Eq. (67) is written as:

$\mathbf{S}^{\mathrm{p}} \Delta \boldsymbol{\sigma}^{e,(n+1)}=\Delta \mathbf{n}^{(n+1)}+\psi^{(n)}$ 
For the subsequent iterations, until $\psi$ becomes arbitrarily small (i.e. $\psi \rightarrow 0$ within some pre-determined tolerance), Eq. (69) is written as:

$\mathbf{S}^{\mathrm{p}} \Delta \boldsymbol{\sigma}^{e,(n+1)}=\psi^{(n+1)}$

In practice, however, only alternative versions of this procedure have been adopted. The most common version, perhaps the most efficient, is that known as the Modified Newton-Raphson (MNR), in which matrix $\mathbf{S}^{\mathbf{p}}$ is updated only once, at the beginning of the iterative process at the beginning of the time-step, and kept unaltered for the subsequent iterations.

(iii) Standard Initial Stress (SIS). This is the explicit case extensively tested; here it is treated as a particular case of (ii) in which matrix $\mathbf{S}^{\mathrm{p}}$ is never updated: $\mathbf{S}^{\mathrm{p}}=\mathbf{I}$.

\section{TD-BEM/D-BEM iterative coupling}

In the present paper, an iterative coupling procedure between the D-BEM and TD-BEM solution algorithms is proposed. It is important to mention that a similar formulation was successfully applied for TD-BEM/FEM iterative coupling [22]. This previous success, of course, encouraged the authors to develop the present formulation, in which one intends to take advantage of the best features of each of the aforementioned BEM formulations.

After one has defined the parts (sub-domains) of the domain to be discretized by the TD-BEM and D-BEM formulations, the following conditions must be satisfied, at each time-step, on the interfaces between sub-domains.

Equilibrium condition:

$\mathbf{p}_{\mathrm{D}}^{(n)}+\mathbf{p}_{\mathrm{TD}}^{(n)}=0$

Continuity condition:

$\mathbf{u}_{\mathrm{D}}^{(n)}-\mathbf{u}_{\mathrm{TD}}^{(n)}=0$

Each sub-domain is solved independently, after prescribing boundary conditions iteratively on the coupling interface, until convergence is reached. This approach looks very attractive, as the systems of equations related to the TDBEM and to the D-BEM can be solved in an independent and uncoupled way.

For greater independence in modelling different subdomains with different formulations, special procedures concerned with interpolation and extrapolation were implemented. As a consequence, different time-steps as well as different spatial discretizations (this means a direct correspondence is not necessary between nodes at common interfaces) can be adopted. Due to this, in order to clarify the concepts involved in the formulation, a modification in the notation is introduced: a discrete time, for instance $t_{n}$, will no longer be represented by the index $(n)$, as in the previous sections; from now on, reference will be made to the time $t_{\mathrm{D}}$ (D-BEM sub-domain) or $t_{\mathrm{TD}}$ (TD-BEM sub-domain).

Initially, the displacements ${ }^{(k+\alpha)} \mathbf{u}_{\mathrm{D}}^{t_{\mathrm{D}}}$, on the sub-domain modelled by the D-BEM, are computed, including the displacements at the interface. The role of the relaxation parameter $\alpha$, as indicated below in Eq. (73), is to guarantee and/or accelerate the convergence of the iterative process; one can write:

${ }^{(k+1)} \mathbf{u}_{\mathrm{D}}^{t_{\mathrm{D}}}=\alpha^{(k+\alpha)} \mathbf{u}_{\mathrm{D}}^{t_{\mathrm{D}}}+(1-\alpha)^{(k)} \mathbf{u}_{\mathrm{D}}^{t_{\mathrm{D}}}$

A study concerning the value to be adopted for the parameter $\alpha$ in the solution of static linear problems with the BEM/FEM coupling can be found in the work by Elleithy et al. [23]. It is to be said that the optimal value of the parameter $\alpha$ is problem-dependent; the parameter can be considered function of the boundary conditions, physical and geometric properties of the sub-domains and the mesh density, etc.

When solving dynamic non-linear problems with TDBEM/D-BEM coupling, according to the authors' experience, generally the choice $\alpha=0.5$ leads to good results, if one thinks about stability (for extreme values, say $\alpha \approx 1.0$, convergence cannot be reached). A more complete discussion concerning the values of this parameter is presented in Section 5, which is related to the numerical examples.

Once the displacements ${ }^{(k+1)} \mathbf{u}_{\mathrm{D}}^{t_{\mathrm{D}}}$ of the D-BEM subdomain have been computed, the displacements ${ }^{(k+1)} \mathbf{u}_{\mathrm{TD}}^{t_{\mathrm{TD}}}$ on the interface can be obtained by means of the continuity condition, given by Eq. (72). As direct correspondence between nodes at the common interface is not necessary, the values of ${ }^{(k+1)} \mathbf{u}_{\mathrm{D}}^{t_{\mathrm{D}}}$ can be interpolated spatially, according to Fig. 1, to obtain ${ }^{(k+1)} \mathbf{u}_{\mathrm{TD}}^{t_{\mathrm{D}}}$. The values of ${ }^{(k+1)} \mathbf{u}_{\mathrm{TD}}^{t_{\mathrm{D}}}$, in its turn, can be extrapolated in time, according to Fig. 2(a), to obtain ${ }^{(k+1)} \mathbf{u}_{\mathrm{TD}}^{t_{\mathrm{TD}}}$. As linear time interpolation was assumed for the displacements in the TD-BEM (Eqs. (19) and (20)), for the time extrapolation one has:

${ }^{(k+1)} \mathbf{u}_{\mathrm{TD}}^{t_{\mathrm{TD}}}={ }^{(k+1)} \mathbf{u}_{\mathrm{TD}}^{t_{\mathrm{D}}} \frac{\Delta t_{\mathrm{TD}}}{\Delta \hat{t}}+\mathbf{u}_{\mathrm{TD}}^{t_{\mathrm{TD}}}-\Delta t_{\mathrm{TD}}\left(1-\frac{\Delta t_{\mathrm{TD}}}{\Delta \hat{t}}\right)$

The TD-BEM sub-domain can, then, be solved: by assuming the known displacements ${ }^{(k+1)} \mathbf{u}_{\mathrm{TD}}^{t_{\mathrm{TD}}}$ as boundary conditions at the coupling interface, the unknowns ${ }^{(k+1)} \mathbf{p}_{\mathrm{TD}}^{t_{\mathrm{TD}}}$ are easily determined.

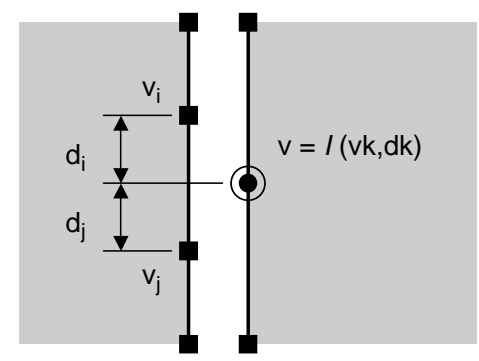

Fig. 1. Spatial interpolation scheme: computing $\mathbf{v}$ from values $\mathbf{v}_{k}$ and $\mathbf{d}_{k}$ at the interface (linear interpolation: $\mathbf{v}=\left(\mathbf{v}_{i} \mathbf{d}_{j}+\mathbf{v}_{j} \mathbf{d}_{i}\right) /\left(\mathbf{d}_{j}+\mathbf{d}_{i}\right)$ ). 
(a)

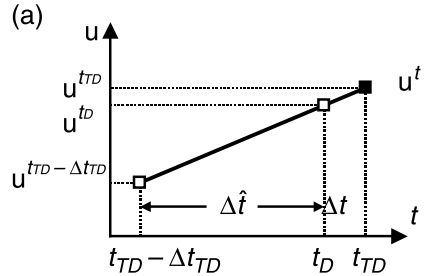

(b)

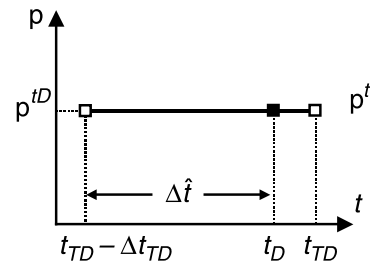

Fig. 2. Time interpolation-extrapolation scheme: (a) time extrapolation from $\mathbf{u}^{t_{\mathrm{D}}}$ to obtain $\mathbf{u}^{t_{\mathrm{TD}}}\left(\mathbf{u}^{t_{\mathrm{TD}}}=\mathbf{u}^{t_{\mathrm{D}}}\left(\Delta t_{\mathrm{TD}} / \Delta \hat{t}\right)+\mathbf{u}^{t_{\mathrm{TD}}-\Delta t_{\mathrm{TD}}}\left(1-\Delta t_{\mathrm{TD}} / \Delta \hat{t}\right)\right) ;(\mathrm{b})$ time interpolation from $\mathbf{p}^{t_{\mathrm{TD}}}$ to obtain $\mathbf{p}^{t_{\mathrm{D}}}\left(\mathbf{p}^{t_{\mathrm{D}}}=\mathbf{p}^{t_{\mathrm{TD}}}\right)$.

The tractions ${ }^{(k+1)} \mathbf{p}_{\mathrm{D}}^{t_{\mathrm{D}}}$ are computed by employing the equilibrium condition, Eq. (66), once the tractions ${ }^{(k+1)}$ $\mathbf{p}_{\mathrm{TD}}^{t_{\mathrm{TD}}}$ have already been computed. When necessary, the values ${ }^{(k+1)} \mathbf{p}_{\mathrm{TD}}^{t_{\mathrm{TD}}}$ can be spatially interpolated, according to Fig. 1, with the purpose of determining ${ }^{(k+1)} \mathbf{p}_{\mathrm{D}}^{t_{\mathrm{T}}}$. The values ${ }^{(k+1)} \mathbf{p}_{\mathrm{D}}^{t_{\mathrm{DD}}}$, in their turn, can be interpolated in time, according to Fig. 2(b) in order to determine ${ }^{(k+1)} \mathbf{p}_{\mathrm{D}}^{t_{\mathrm{D}}}$. As the tractions are assumed to have constant variation along time in the TD-BEM formulation (Eqs. (21) and (22)), for the time interpolation discussed in the present section one has:

${ }^{(k+1)} \mathbf{p}_{\mathrm{D}}^{t_{\mathrm{D}}}={ }^{(k+1)} \mathbf{p}_{\mathrm{D}}^{t_{\mathrm{DD}}}$

After computing ${ }^{(k+1)} \mathbf{p}_{\mathrm{D}}^{t_{\mathrm{D}}}$ it becomes necessary to verify the convergence of the iterative process. Once convergence is achieved, the variables are updated and the analysis begins for the next time-step.

It is important to point out the reduced computational cost of the iterative coupling: the convergence is fast and the coupling iterations are performed together with the iterations of the non-linear problem. A positive feature of the proposed methodology is related to the solution of the TD-BEM and D-BEM sub-domains: as the solutions are carried out separately, smaller and better conditioned systems of equations are obtained, providing efficient analyses and accurate results.

Another positive feature is the possibility of carrying out analyses with different time-steps for the various subdomains. As mentioned before, the correct choice of a time-step plays a fundamental role in BEM analyses. In general, the time-steps required by the TD-BEM formulation are greater than the time-steps required by the D-BEM formulation; neglecting this fact can lead to unstable coupling algorithms.

\section{Numerical examples}

Two examples are considered: the first one deals with a linear one-dimensional rod (finite-domain problem); and the second one is concerned with a non-linear circular cavity (infinite-domain problem). Results related to the proposed TD-BEM/D-BEM coupling algorithm are compared with analytical results, whenever possible, or with other numerical results, showing the good accuracy of the proposed methodology.

\subsection{One-dimensional rod}

This is a classical example and is included here to verify the potentialities of the proposed formulation. The example consists of a one-dimensional rod submitted to a Heaviside type forcing load applied at $t=0 \mathrm{~s}$ and kept constant in time [9,14,21]. Fig. 3(a) shows the boundary conditions, geometry and selected internal point A. Fig. 3(b) shows the coupled TD-BEM/D-BEM meshes adopted: in coupled mesh 1, 64 linear boundary elements of equal length are employed (32 boundary elements for TD-BEM and 32 boundary elements for D-BEM), as well as 128 linear triangular integration cells (D-BEM formulation); in coupled mesh 2, 48 linear boundary elements are employed (16 boundary elements, of equal length, for TD-BEM and 32 boundary elements, of equal length, for D-BEM). The physical properties of the rod are given by $\nu=0.0$ (in order to simulate a one-dimensional analysis); $E=100 \mathrm{~N} / \mathrm{m}^{2} ; \rho=$ $1.5 \mathrm{~kg} / \mathrm{m}^{3}$. The geometry of the rod is defined by $a=2 \mathrm{~m}$ and $b=1 \mathrm{~m}$.

In Figs. 4 and 5, traction time histories at point $\mathrm{A}(a$ / $2 ; b / 2$ ) are plotted considering coupled meshes 1 and 2, respectively, for different time steps in each sub-domain. In Table 1, the average number of iterations versus time

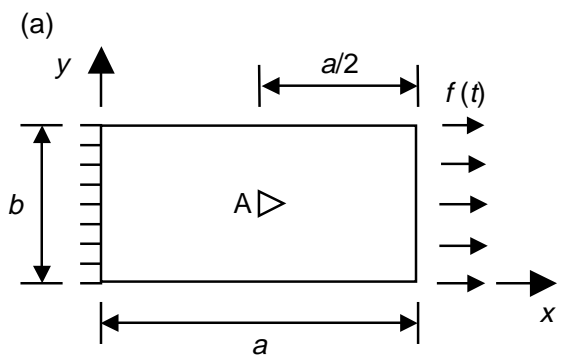

(b)

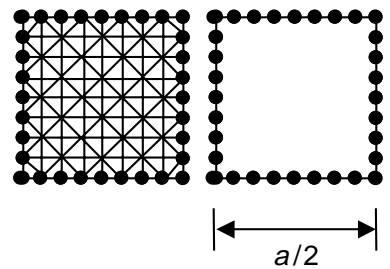

(c)

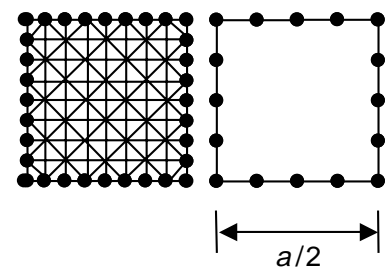

Fig. 3. One dimension rod: (a) geometry, boundary conditions and selected internal point; (b) coupled mesh 1; (c) coupled mesh 2. 

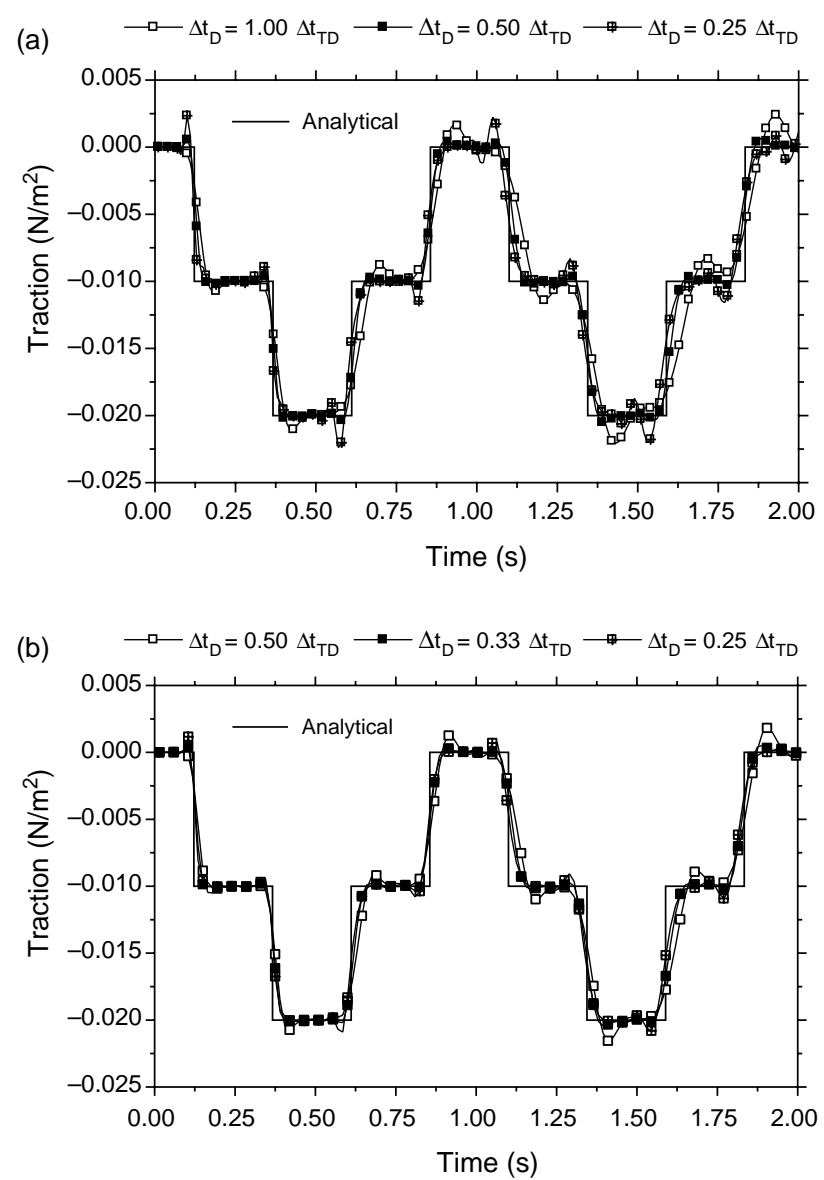

Fig. 4. Horizontal traction at point $\mathrm{A}$ considering coupled mesh 1 and different time discretizations: (a) $\Delta t_{\mathrm{TD}}=0.01 \mathrm{~s}\left(\beta_{\mathrm{TD}} \approx 0.65\right)$; (b) $\Delta t_{\mathrm{TD}}=0$. $015 \mathrm{~s}\left(\beta_{\mathrm{TD}} \approx 1.00\right)$.

step value for the TD-BEM/D-BEM iterative coupling is shown, as a function of the relaxation parameter $\alpha$. The results depicted in Figs. 4 and 5 are obtained with $\alpha=$ 0.5 . For $\alpha=1.0$ and $\Delta t_{\mathrm{TD}}=\Delta t_{\mathrm{D}}=0.01 \mathrm{~s}$ (coupled meshes 1 and 2), as well as for $\Delta t_{\mathrm{TD}}=2 \Delta t_{\mathrm{D}}=0.015 \mathrm{~s}$ (coupled mesh 1), the iterative coupling procedure becomes unstable. The results depicted in Table 1 were obtained adopting a node-by-node convergence check for displacements and tractions along the coupling interface, with tolerance $10^{-3}$.

As one can observe, the proposed methodology is accurate (adopting an appropriate time/space-discretization relationship) and efficient (adopting an appropriate $\alpha$ value).

\subsection{Circular cavity}

This plane strain problem consists of a circular cavity under a uniform internal pressure suddenly applied and kept constant in time $[9,21]$. A sketch of the model is shown in Fig. 6(a). The boundary element and internal cell discretization are depicted in Fig. 6(b): 46 linear boundary
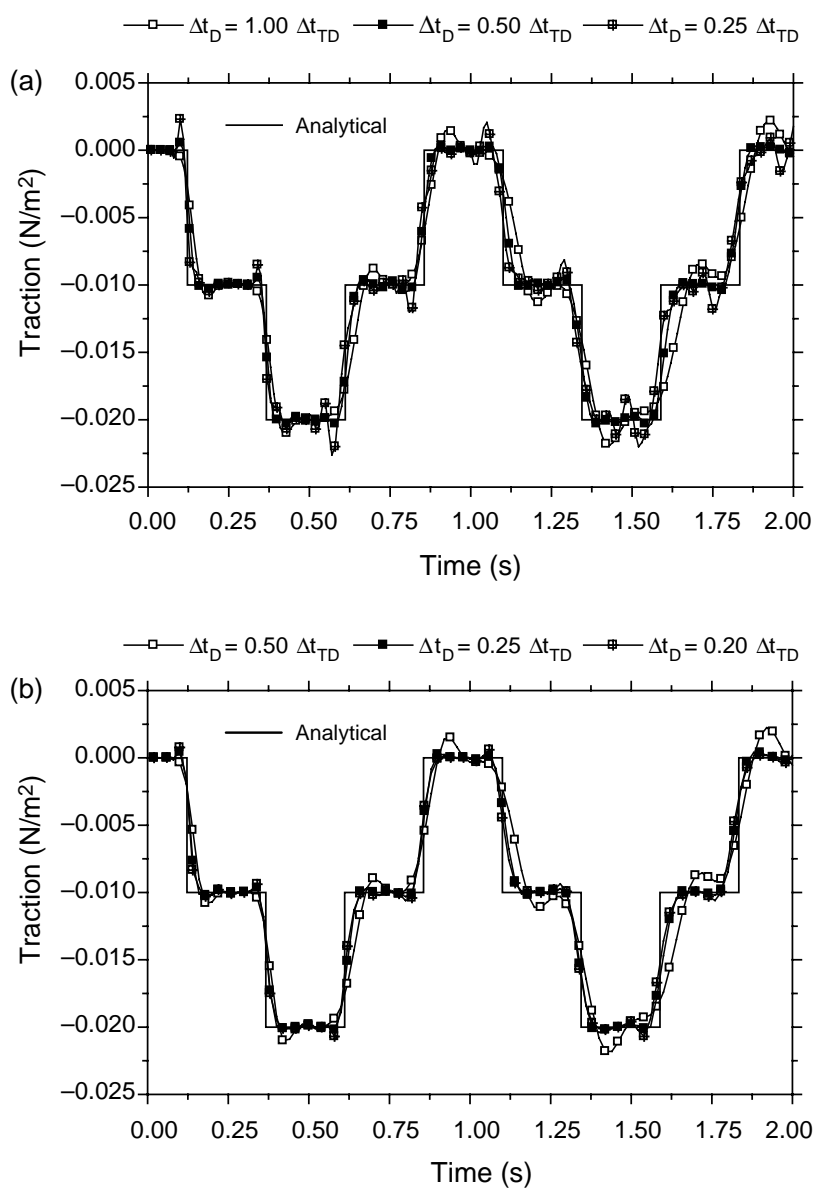

Fig. 5. Horizontal traction at point A considering coupled mesh 2 and different time discretizations: (a) $\Delta t_{\mathrm{TD}}=0.01 \mathrm{~s}\left(\beta_{\mathrm{TD}} \approx 0.33\right)$; (b) $\Delta t_{\mathrm{TD}}=0$. $02 \mathrm{~s}\left(\beta_{\mathrm{TD}} \approx 0.65\right)$.

elements are employed in the TD-BEM/D-BEM coupled analysis (20 boundary elements for TD-BEM and 26 boundary elements for D-BEM), as well as 270 linear triangular cells (D-BEM formulation). In the present analysis the double symmetry of the problem is taken into account. An interesting feature of the boundary element formulation is that symmetric bodies under symmetric loads can be analysed without discretization of the symmetry axes. This can be accomplished by an automatic condensation process, which integrates over reflected elements and performs the assemblage of the final matrices in reduced size [1].

The physical properties of the model are: $\nu=0.2308$; $E=6.5277 \times 10^{8} \mathrm{~N} / \mathrm{m}^{2} ; \rho=1.804 \times 10^{3} \mathrm{~kg} / \mathrm{m}^{3}$. A perfectly plastic material obeying the Mohr-Coulomb yield criterion is assumed: cohesion $4.8263 \times 10^{6} \mathrm{~N} / \mathrm{m}^{2}$; internal friction angle $30^{\circ}$. The geometry of the problem is defined by: $R=3.048 \mathrm{~m} ; d=3.658 \mathrm{~m}$. The time discretization adopted is given by: $\Delta t_{\mathrm{TD}}=\Delta t_{\mathrm{D}}=0.2 \mathrm{~s}$ $(\beta \approx 0.285)$.

Time histories of radial $\left(\sigma_{\mathrm{R}}\right)$ and circumferential $\left(\sigma_{\mathrm{C}}\right)$ stress components, at boundary node $\mathrm{A}(x=R$ and $y=0)$ 
Table 1

Average number of iterations per time step

\begin{tabular}{|c|c|c|c|c|c|c|c|}
\hline \multirow{2}{*}{$\begin{array}{l}\Delta t_{\mathrm{TD}}(\mathrm{s})= \\
\Delta t_{\mathrm{D}}(\mathrm{s})=\end{array}$} & \multicolumn{3}{|l|}{0.01} & \multicolumn{3}{|l|}{0.015} & \multirow{2}{*}{$\begin{array}{l}\text { Relaxation } \\
\text { parameter }\end{array}$} \\
\hline & 0.01 & 0.005 & 0.0025 & 0.0075 & 0.005 & 0.00375 & \\
\hline \multirow[t]{4}{*}{ Coupled mesh 1} & 12 & 12 & 13 & 12 & 12 & 13 & 0.25 \\
\hline & 5 & 6 & 7 & 6 & 6 & 6 & 0.50 \\
\hline & 7 & 4 & 4 & 5 & 4 & 4 & 0.75 \\
\hline & $\mathrm{X}$ & 7 & 3 & $\mathrm{X}$ & 17 & 6 & 1.00 \\
\hline$\Delta t_{\mathrm{TD}}(\mathrm{s})=$ & \multicolumn{3}{|l|}{0.01} & \multicolumn{3}{|l|}{0.02} & \multirow{2}{*}{$\begin{array}{l}\text { Relaxation } \\
\text { parameter }\end{array}$} \\
\hline$\Delta t_{\mathrm{D}}(\mathrm{s})=$ & 0.01 & 0.005 & 0.0025 & 0.01 & 0.005 & 0.004 & \\
\hline \multirow[t]{4}{*}{ Coupled mesh 2} & 12 & 13 & 13 & 14 & 13 & 13 & 0.25 \\
\hline & 6 & 6 & 7 & 6 & 6 & 7 & 0.50 \\
\hline & 7 & 4 & 4 & 6 & 4 & 4 & 0.75 \\
\hline & 63 & 7 & 3 & $\mathrm{X}$ & 8 & 5 & 1.00 \\
\hline
\end{tabular}

(a)

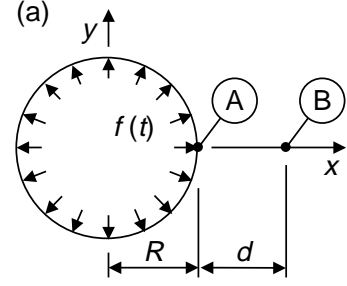

(b)

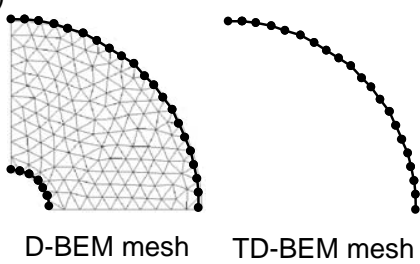

Fig. 6. Circular cavity: (a) geometry, boundary conditions and selected boundary node and internal point; (b) coupled mesh.
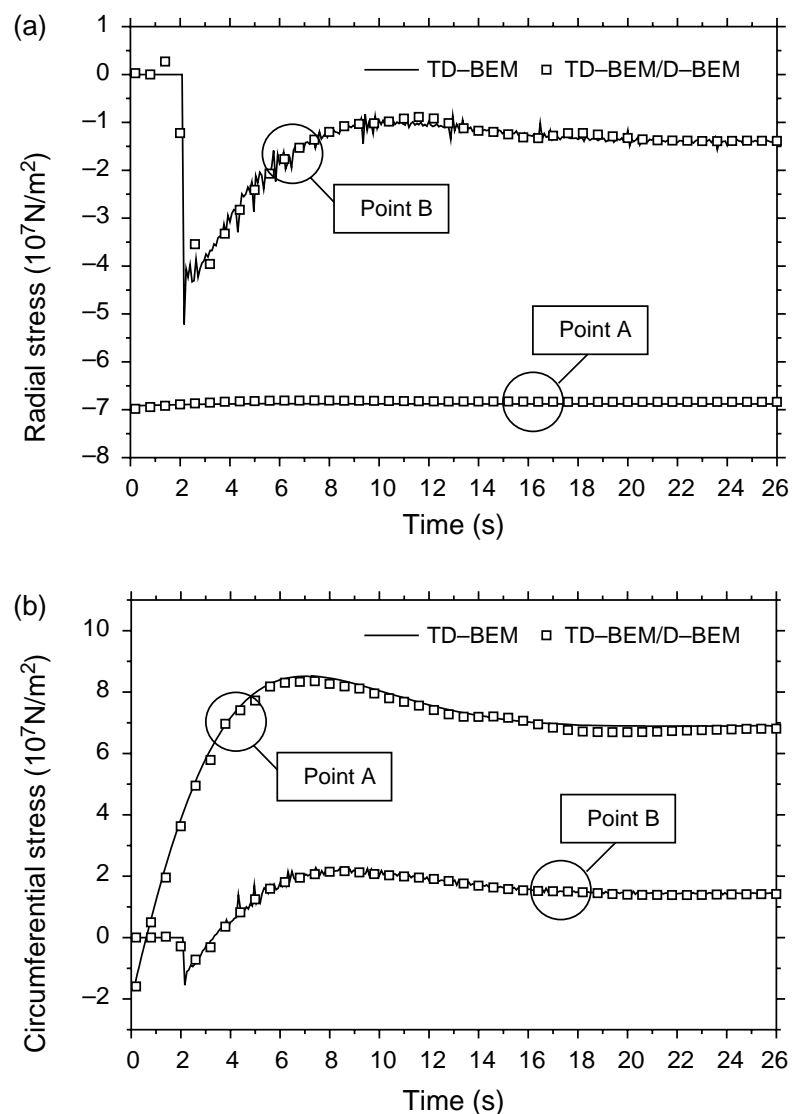

Fig. 7. Linear stresses: TD-BEM solution and coupled TD-BEM/D-BEM solution: (a) $\sigma_{\mathrm{R}}$, radial stress; (b) $\sigma_{\mathrm{C}}$, circumferential stress.

and internal point $\mathrm{B}(x=R+d$ and $y=0)$, are depicted in Figs. 7 and 8. Displacement time history is plotted in Fig. 9. The linear TD-BEM/D-BEM results are compared with results related to the TD-BEM formulation, obtained by adopting a richer mesh of 12 linear boundary elements to model the $1 / 4$ of the cavity (once again the symmetry of the problem is considered). Stresses at internal points for the TD-BEM analysis were computed employing complex algebra as indicated by Soares Jr. et al. [21]. As one can observe, good
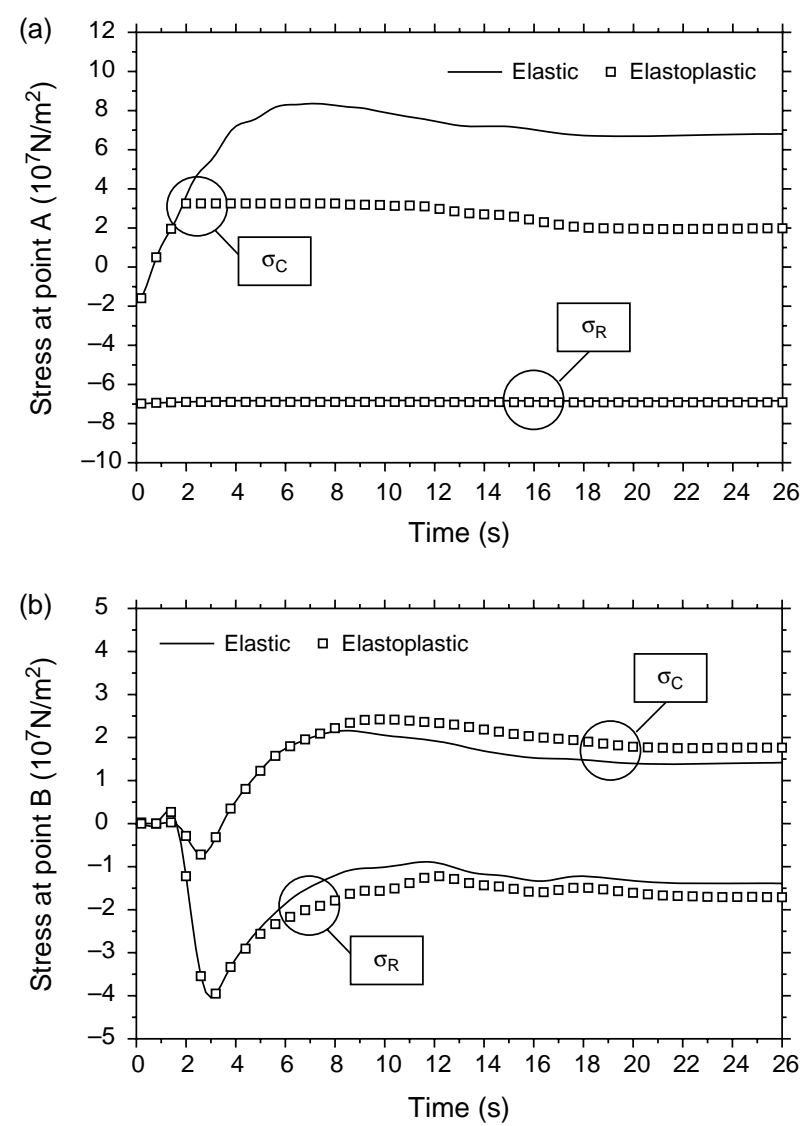

Fig. 8. Linear and non-linear stresses: coupled TD-BEM/D-BEM solution: (a) stresses at point A; (b) stresses at point B. 

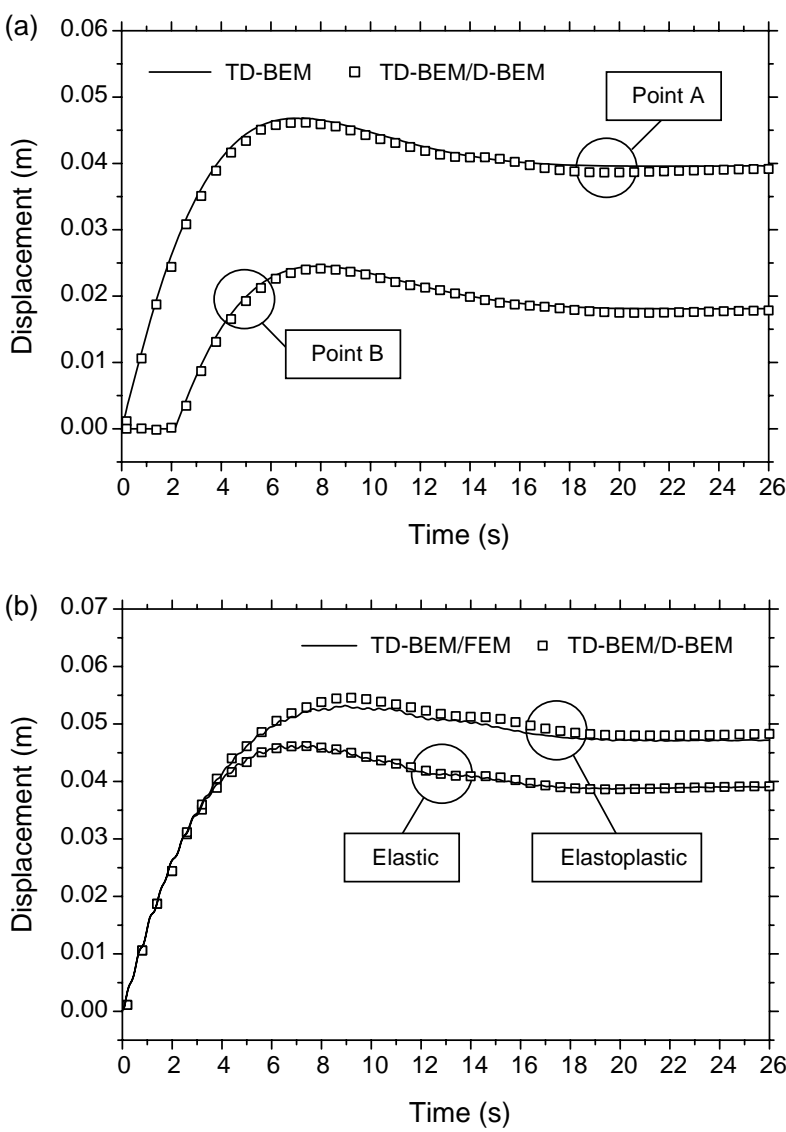

Fig. 9. Radial displacement considering TD-BEM, coupled TD-BEM/DBEM, and coupled TD-BEM/FEM solutions: (a) linear results; (b) linear and non-linear results at point $\mathrm{A}$.

agreement is obtained between the TD-BEM and TDBEM/D-BEM formulations (Figs. 7-9 are also in good agreement with results obtained by Chow and Koenig [24]).

In Fig. 9(b), the TD-BEM/D-BEM displacement time history at point $\mathrm{A}$ is plotted, considering linear and nonlinear analyses. Results concerning a TD-BEM/FEM coupled analysis [25] are also plotted in Fig. 9(b); once again, good agreement can be observed.

\section{Conclusions}

The methodology for the solution of non-linear dynamic problems presented in this work proved to be very efficient, as it makes use of the main advantages of two different BEM formulations: finite domains with non-linear behaviour can be modelled by the D-BEM formulation, which is very simple, and infinite (finite) domains with linear behaviour can be modelled by the TD-BEM formulation. Due to the characteristics of the latter, it is possible to solve infinite domain problems very appropriately, since no artificial boundaries are introduced in the analysis (this eliminates the possible influence of spurious reflected waves). Besides, iterative coupling at common interfaces does not demand a high computational cost and can be easily implemented in a computer code. Accurate results were obtained, as demonstrated by the two examples included here, encouraging further developments, e.g. iterative coupling between TD-BEM and DR-BEM formulations and the extension for 3-D problems.

\section{References}

[1] Telles JCF. The boundary element method applied to inelastic problems. Lecture notes in engineering. vol. 1. Berlin: Springer; 1983.

[2] Carrer JAM, Telles JCF. A boundary element formulation to solve transient dynamic elastoplastic problems. Comput Struct 1992;45: 707-13.

[3] Kontoni DPN, Beskos DE. Transient dynamic elastoplastic analysis by the dual reciprocity BEM. Eng Anal Bound Elem 1993;12:1-16.

[4] Partridge PW, Brebbia CA, Wrobel LC. The dual reciprocity boundary element method. Southampton: Computational Mechanics Publications; 1992.

[5] Houbolt JC. A recurrence matrix solution for the dynamic response of elastic aircraft. J Aeronaut Sci 1950;17:540-50.

[6] Carrer JAM, Mansur WJ. Alternative time-marching schemes for elastodynamic analysis with the domain boundary element method formulation. Comput Mech 2004;34:387-99.

[7] Souza LA, Carrer JAM, Martins CJ. A fourth order finite difference method applied to elastodynamics: finite element and boundary element formulations. Struct Eng Mech 2004;17:735-49.

[8] Eringen AC, Suhubi ES. Elastodynamics. vols. I and II. New York: Academic Press; 1975.

[9] Mansur WJ. A time-stepping technique to solve wave propagation problems using the boundary element method. PhD Thesis. University of Southampton, England; 1983.

[10] Dominguez J. Boundary elements in dynamics. Southampton: Computational Mechanics Publications; 1993.

[11] Soares Jr D, Mansur WJ. Compression of time generated matrices in two-dimensional time-domain elastodynamic BEM analysis. Int J Numer Meth Eng 2004;61:1209-18.

[12] Hadamard J. Lectures on Cauchy's problem in linear partial differential equations. New York: Dover; 1952.

[13] Mansur WJ, Carrer JAM. Two-dimensional transient BEM analysis for the scalar wave equation: kernels. Eng Anal Bound Elem 1993;12: 283-8.

[14] Carrer JAM, Mansur WJ. Stress and velocity in 2D transient elastodynamic analysis by the boundary element method. Eng Anal Bound Elem 1999;23:233-45.

[15] Bathe KJ. Finite element procedures. Englewood Cliffs, NJ: PrenticeHall; 1996.

[16] Weaver Jr W, Johnston PR. Structural dynamics by finite elements. Englewood Cliffs, NJ: Prentice-Hall; 1987.

[17] Hatzigeorgiou GD, Beskos DE. Transient Dynamic Response of 3D Elastoplastic Structures by the D/BEM. In: Beskos DE, Brebbia CA, Katsikadelis JT, Manolis GD, editors. Proceeding of boundary elements XXIII, Lemnos, Greece.

[18] Newmark NM. A method of computation for structural dynamics. ASCE J Eng Mech Div 1959;85:67-94.

[19] Lyness JN, Moler CB. Numerical differentiation of analytic functions. SIAM J Numer Anal 1967;4:202-10.

[20] Gao XW, Liu DD, Chen PC. Computation of internal stresses in nonlinear BEM using a numerically-exact complex-variable approach. In: Denda M, Aliabadi MH, Charafi A, editors. Proceeding of advances in boundary element techniques II, Rutgers. The State University of New Jersey; 2001 
[21] Soares Jr. D, Carrer JAM, Telles JCF, Mansur WJ. Time-domain BEM formulation: two approaches for the computation of stress and velocity. Comput Mech 2002;30:38-47.

[22] Soares Jr D, von Estorff O, Mansur WJ. Iterative coupling of BEM and FEM for nonlinear dynamic analysis. Comput Mech 2004;34:67-73.

[23] Elleithy WM, Al-Gahtani HJ, El-Gebeily M. Iterative coupling of BE and FE methods in elastostatics. Eng Anal Bound Elem 2001;25: $685-95$.
[24] Chow PC, Koenig HA. A unified approach to cylindrical and spherical elastic waves by method of characteristics. Trans ASME, J Appl Mech 1966;33(Series E):159-67.

[25] Soares Jr D. Dynamic analysis of non-linear soil-fluid-structure coupled systems by the finite element method and the boundary element method (in Portuguese). PhD Thesis. Federal University of Rio de Janeiro, Brazil; 2004. 